\title{
QPSK Demodulator Based on Wideband Acquisition System
}

\author{
Kokate M. D., Abhay E. Wagh, Wankhede V. A.
}

\begin{abstract}
The paper describes the design of the QPSK demodulator based satellite base station. The most important requirement of the design process is to have wide band acquisition range of $100 \mathrm{kHz}$ under narrow Phase Lock Loop (PLL) bandwidth and low input Signal to Noise Ratio (SNR). The efficiency of the technique is verified with extensive simulations in MATLAB.
\end{abstract}

Keywords: About four key words or phrases in alphabetical order, separated by commas.

\section{INTRODUCTION}

Satellite is used to transmit any kind of digital data to the earth station. The data can be a video signal, an audio signal or any digital data which is collected from various cosmic sensors. During the transmission of the signal, satellite is continuously revolving around the earth. Due to this continuous movement of the satellite, the satellite communication gets affected by the DOPPLER EFFECT. Due to this Doppler Effect, the Doppler shift occurs in the communication. Because of this the original transmissions of the signal get some frequency shift. As satellite is used for the transmission of critical information and if this Doppler Effect occurs then the information loss takes place and this is not affordable. So that a need exists to develop a satellite base station which is capable to overcome this problem. This can be done by providing the wideband for reception. And in reception, this wideband is provided by using phase lock loop.

\section{A. Proposed enhanced system}

The design of the wideband acquisition technique for a demodulator is an important problem in satellite communication, because of the Doppler Effect. The Doppler shift for the downlink signal from the low-earth orbit satellites as on earth is in the range around $100 \mathrm{kHz}$ and also the telemetry downlink data rates are variable. Hence, a need exists for development of ground based configurable receiver to support these modulation formats for variable data rates and support the wideband acquisition range. The PLL based receivers can lock onto incoming signal without cycle slip to a bandwidth of $2.8 \times B L$, where $\mathrm{BL}$ is the bandwidth of the loop filter. If the bandwidth increases, the signal to noise ratio will be decreased.

Revised Manuscript Received on February 05, 2020

* Correspondence Author

M. D. Kokate, Principal, SNJB's College of Engineering Chandwad, MS, India.

Dr. Abhay Wagh, Dy. Director and Assistant Director, Directorate of Technical Education, Maharashtra, India.

Dr. Wankhede Vishal Ashok, Principal, SNJBs S.H.H.J.B. Polytechnic Chandwad, India.

(c) The Authors. Published by Blue Eyes Intelligence Engineering and Sciences Publication (BEIESP). This is an open access article under the CC BY-NC-ND license (http://creativecommons.org/licenses/by-nc-nd/4.0/)
Hence, to ensure wideband acquisition within the predefined acquisition time, several methods are devised. Basically, the variable loop bandwidth acquisition and aided acquisition techniques are used to perform wideband acquisition in PLL designs.

\section{BLOCK DIAGRAM OF QPSK DEMODULATOR BASED ON WIDEBAND ACQUISITION TECHNIQUE.}

Fig.(1) shows the MATLAB simulink model block diagram of the system. The figure is simulation model of satellite transmitter as well as the satellite receiver. The model is configured as per the parameter required for the actual satellite communication. The model is implemented and tested in MATLAB. Satellite uses any kind of signal for transmission of the signal, it can be audio signal, video signal or it may be a digital data which is collected from the various sensors. The collected digital data is first converted into the QPSK modulated data. After the QPSK modulation, the digital modulating signal is placed in the seperate quadrature.The QPSK transmitted signal consist of data rate of 10 mbps with $10 \mathrm{mHz}$ of frequency. Further this modulating signal is given to the upconversion block. Here the modulating signal is upconverted upto $100 \mathrm{MHZ}$ frequency.Then this upconverted signal is passed through the channel. In every communication system, channels play a very crutial role. In channel doppler shift along with doppler shift adaptive white gaussian noise also get added with the original modulating signal.The channel is configured as per original paramenter similar to the wireless channel of satellite communication. Before giving the signal to the channel,a band is provided to the signal. Till now this blocks are acting as the satellite transmitter. Now this modulated signal is given to the downconversion block. As in transmitter section the signal is upconverted,it is also necessary to downconvert this upconverted signal. This is done by the downconversion block. Also this downconversion block seperate out the real and imaginary part present in the signal.Then both the signals are given to the baseband signal receiver.This receiver consist of the phase lock loop which is the main part of the system. The role of the phase lock loop is to provide the widedband as transmitted signal has. Due to this, the signal is received in between provided wideband and it causes reduction in the doppler effect and the frequency and phase shift in the reception of signal.

\section{A. Signal Source}

Satellite is used for the transmission of data. 


\section{QPSK Demodulator Based on Wideband Acquisition System}

Data can be a video signal, an audio signal or it can be a digital signal which are coming from the various sensors.

The role of the satellite is to collect this signals, modulate it, upconvert this signals and transmitt it towards the satellite reception earth base station.Generally satellite uses the QPSK modulation scheme. The data is first converted into the packets of QPSK, this could be done by the MATLAB code which is shown in Fig.(2) And then this packets act as the signal source. An easy way to comply with the paper formatting requirements is to use this document as a template and simply type your text into it.

\section{B. Upconversion stage}

In telecommunication, transmitting the modulating signal to long distance receiver directly is not possible because the signal gets atteneuted. So that there are two techniques therotically available that are either increase the height of the anttena in certain kilometers or second one is to upconvert the modulating signal frequency.

But practically implementation of the antennas with certain kilometers of height is not possible so that the second method i.e. upconversion of the signal is used. Here, we are using the same technology, the modulating signal has the frequency of $10 \mathrm{MHz}$ which is applied to the upconversion product of modulating signal and signal generated by the local oscillator. Fig.(3) shows the internal block diagram of the upconversion block.

Upconversion block get the signal which has the frequency of $10 \mathrm{MHz}$ then this signal is applied to the sample and hold block which provides the samples of the input signal with the rate of $1 / \mathrm{T}$ and the value of $\mathrm{T}$ is $0.1 \mu \mathrm{sec}$, due to this, we get 10 mbps at the output of sample and hold block. Further, this signal is provided to the square cosine filter. Square cosine filter increases the symbol period. Here the sample period is doubled using the square cosine filter.

Now this signal is applied to the product block in which the actual upconversion of signal takes place. Product block has two inputs first one from the output of sqaure cosine filter and the second one is from the local oscillator. The local osciallator is generating the frequency at rate of $1 / \mathrm{Tc}$ where the value of $\mathrm{Tc}=0.01 \mu \mathrm{sec}$ i.e we are getting total of $100 \mathrm{MHz}$ frequency at the output of the local oscillator. This frequency is applied to the product block along with the modulating signal and product of both signals is taken there.As a output of the product block we are getting upconverted frequency signal which has the frequency of $100 \mathrm{MHz}$. Similar process is done for the imaginary part also, and at last both the real and imaginary parts are combined and forwarded to the channel.

\section{AWGN channel}

The internal block diagram of the upconversion is shown in Fig.(4). The upconverted signal is given to the band pass AWGN channel. This channel is configured as per the communication channel which is similar to the satellite communication channel. In this block a input signal is provided with a band of $90 \mathrm{MHz}$ starting from the frequency block which converts this $10 \mathrm{MHz}$ into $100 \mathrm{MHz}$ by taking

of $25 \mathrm{MHz}$ to $115 \mathrm{MHz}$. Due to this, only frequency signals which are in between this band are allowed to pass through bandpass filter and this signal further get sampled at a rate of Tc/10 i.e $0.01 \mu / 10$ that of 0.001 samples per second. Along with this, the main part of the system that is the doppler shift is also added with the signal. Here the total of $30 \mathrm{kHz}$ doppler shift is added to the system. In satellite communication, not only the doppler effect but various noises are also added with original transmitter signal. For this, we are using the AWGN channel which consist of adaptive white guassian noise which is random in nature.

\section{Down conversion}

The down conversion block is shown in Fig. (5). Once the signal is passed from the AWGN noise channel, this signal is given to the Down conversion block. In transmitter section the modulating signal is up converted up to $100 \mathrm{MHz}$ by adding the external frequency signal coming from the local oscillator. The local oscillator frequency signal does not contain any kind of the information; they are just used for up conversion process. Due to which in receiver section it is important to separate out the information and the carrier which are added in down conversion process. This is achieved by using the down conversion block. The down conversion is the similar process like the up conversion but the main difference is that, in down conversion the carrier signals are removed from the modulated signal and this is done with the help of similar local oscillator which has same frequency of operation as in transmitter section. This frequency signals are subtracted from modulated signal and at the end of the down conversion stage we get the down converted signal.

\section{E. Phase lock loop}

Fig.(6) shows the block diagram of the phase lock loop.The down converted signal consist of the both real and imaginary part of the information, which is applied to the phase recovery loop, in which the phase lock loop is used. The main role of the phase lock loop is to provide a wideband to the receiver so that it can overcome from the problem of the doppler effect. Phase lock loop has three stages, the first stage is free running stage. In free running stage phase lock loop generates the output as similar to the received along with this, it gives some part of the signal to the QPSK phase error detector. The second stage of the phase lock loop is processing stage. In processing stage, phase lock loop estimate the errors that are received and depending upon the error detected the corrective action is taken and passed to the input. This is done with the help of feedback system. This action takes places until the error gets zero value. And the third stage is lock stage. In lock stage, the error becomes zero due to which phase lock loop locks the received frequency and the frequency which is desired frequency. The diffrence between this two frequencies is called as the wideband. Due to this wideband acquisition even though their is doppler shift in signal,it is removed in phase lock loop . 
The action of locking frequency and phase is taken for both the parts of signal i.e for real as well as imaginary part of the signal. In this system, phase lock loop plays an important role. It provides the wideband starting from $25 \mathrm{MHz}$ and ending at $115 \mathrm{MHz}$ i.e total of $90 \mathrm{MHz}$. Due to this wideband, the data acquisition of signal takes place effectively and this also provides the proper reception of the signal, eventhough the received signal is effected by the doppler shift.
Due to this provided wideband of the phase lock loop, the receiver is capable of receiving this signal properly and remove the doppler shift.

As a result to this, we receive the costellation of the signal in proper quadrants as per the transmitted quadrants.

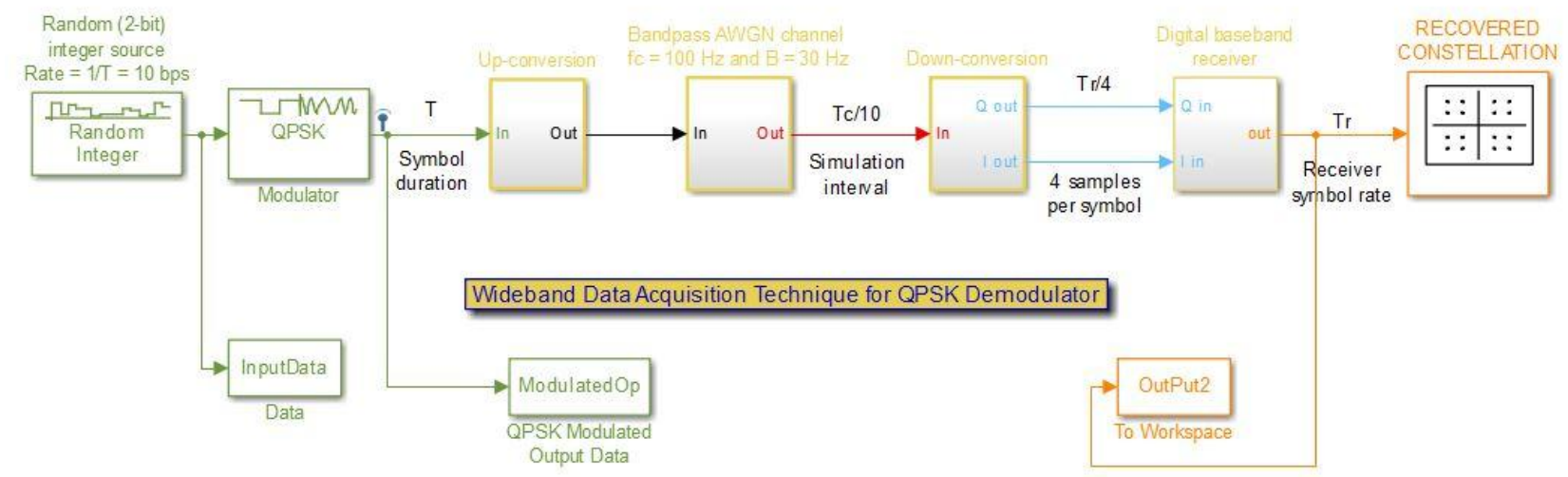

Fig. (1) Wideband Data Acquisition Technique for QPSK Demodulator.
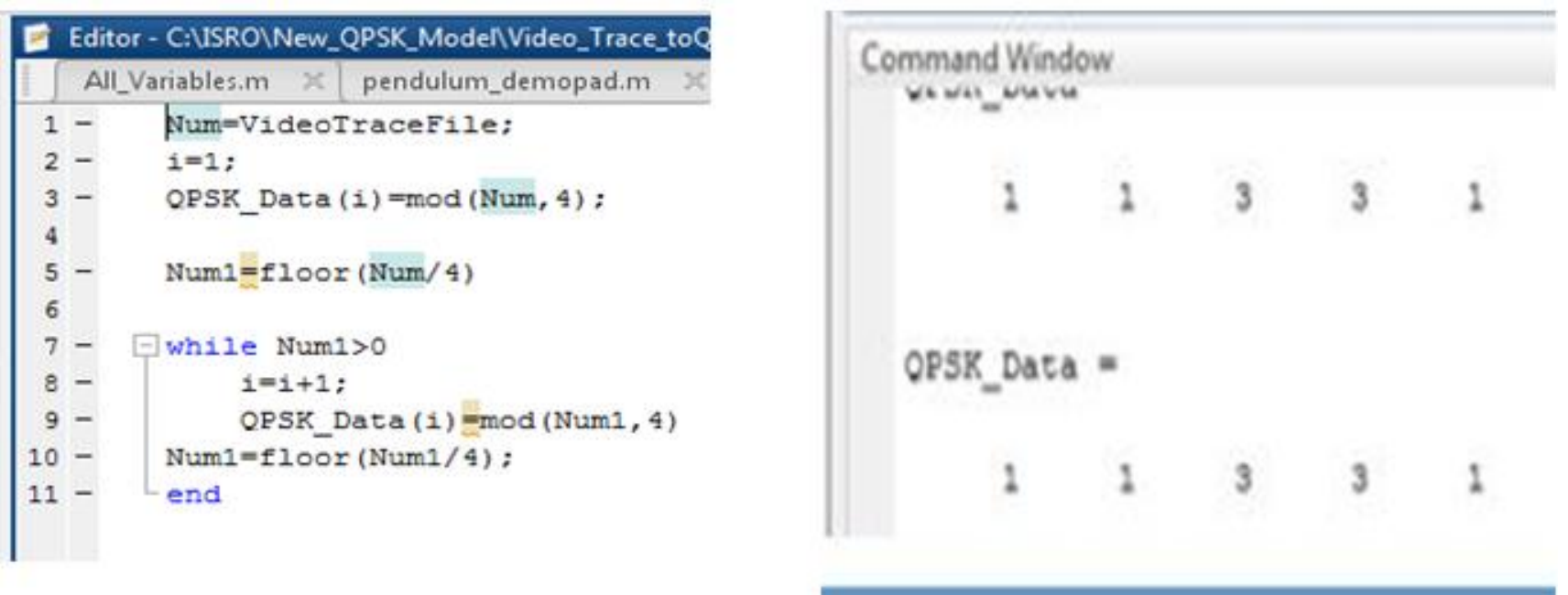

Fig. (2) QPSK packet formation program.

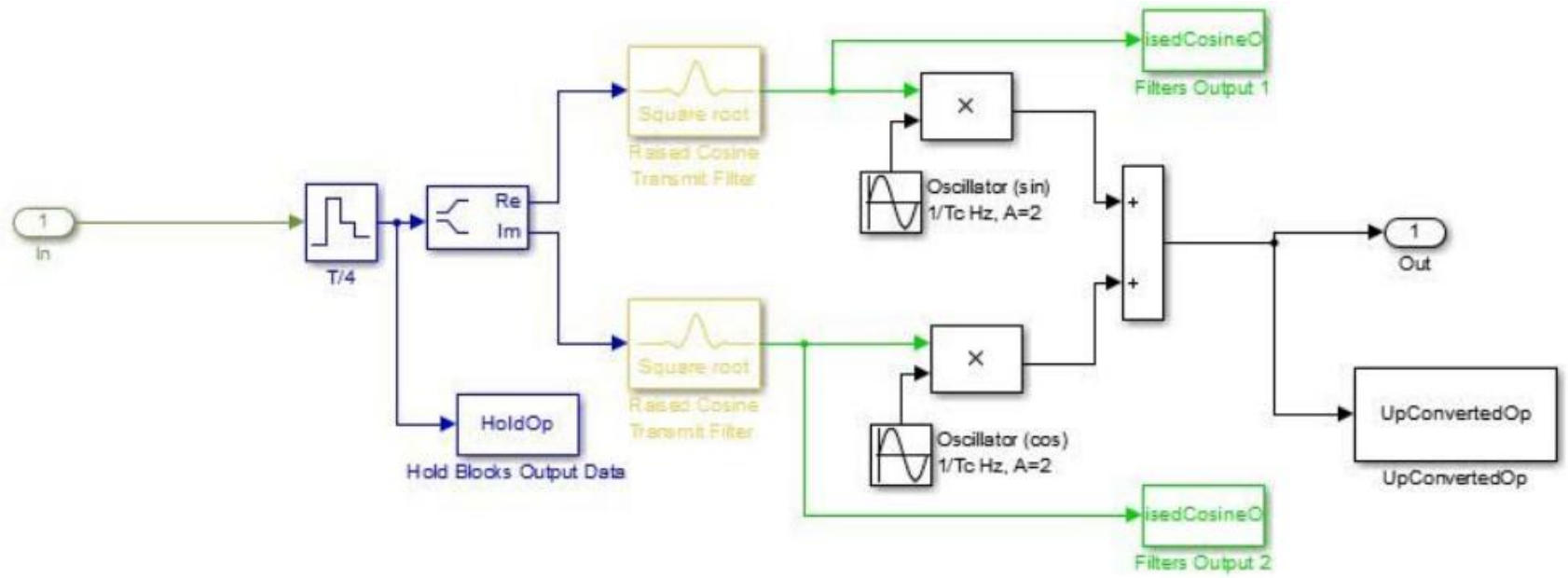

Fig. (3) Up conversion stage 


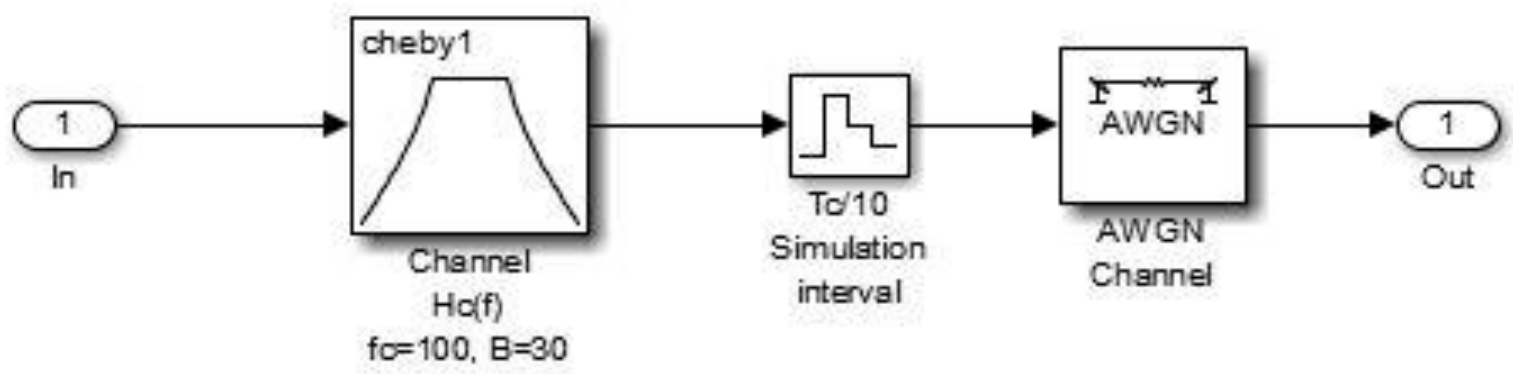

Fig. (4) AWGN Channel.

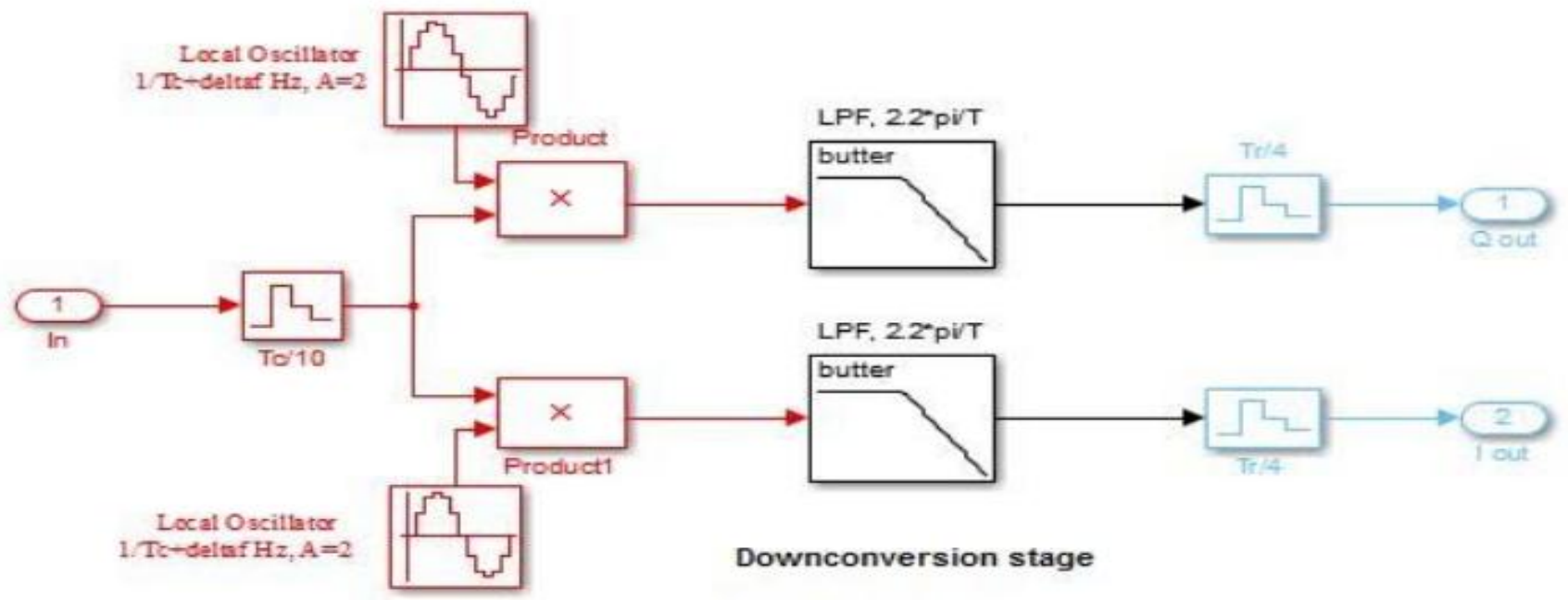

Fig. (5) Down conversion Stage

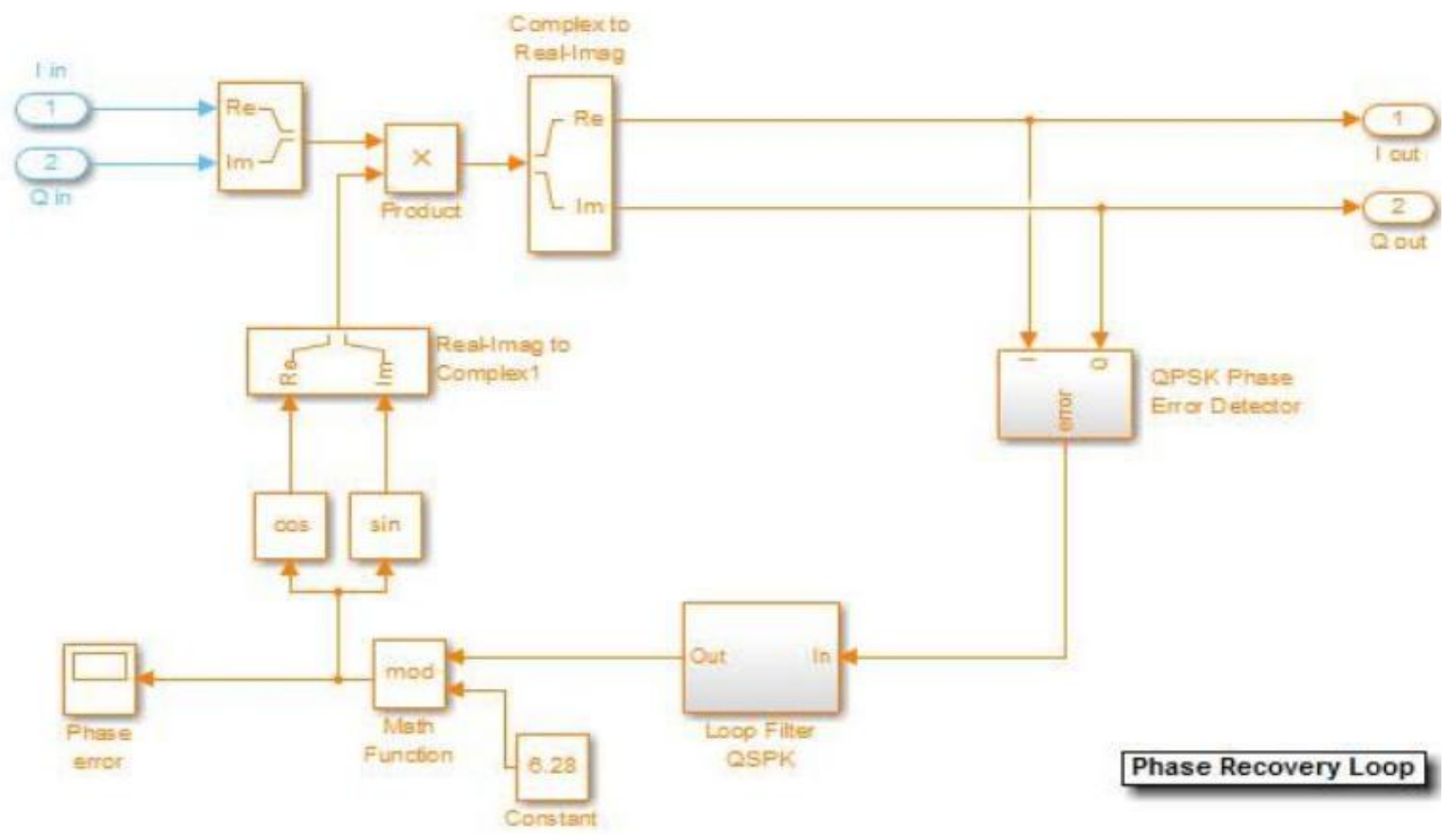

Fig. (6) Phase Lock Loop

Retrieval Number: C4847029320/2020@BEIESP

\section{Published By:}




\section{EXPERIMENTAL ANALYSIS AND RESULT}

A. Constellation diagram before using the phase lock loop

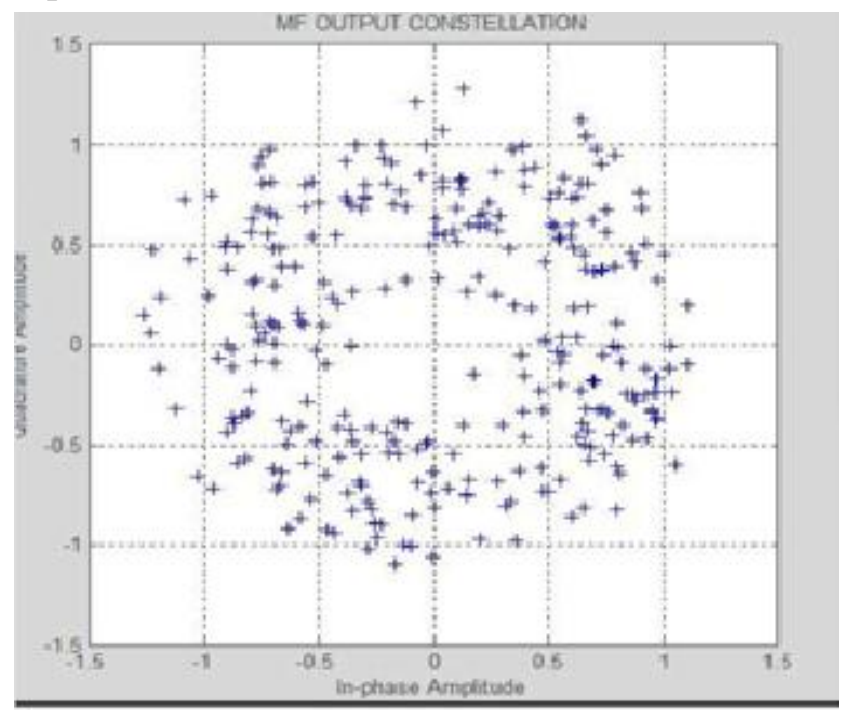

Fig. (7) Constellation diagram without phase lock loop

Fig.(7) shows the constellation diagram of received signal. As shown in figure, when there is no phase lock loop the constellation of the received signal are scattered in all quadrants due to which it becomes more complex to identify which signal lies in which quadrant.

\section{B. Constellation diagram after using the phase lock.}

A carrier recovery system is a circuit used to estimate and compensate for frequency and phase differences between a received signal's carrier wave and the receiver's local oscillator for the purpose of coherent demodulation. In the transmitter of a communications carrier system, a carrier wave is modulated by a baseband signal. At the receiver the baseband information is extracted from the incoming modulated waveform.

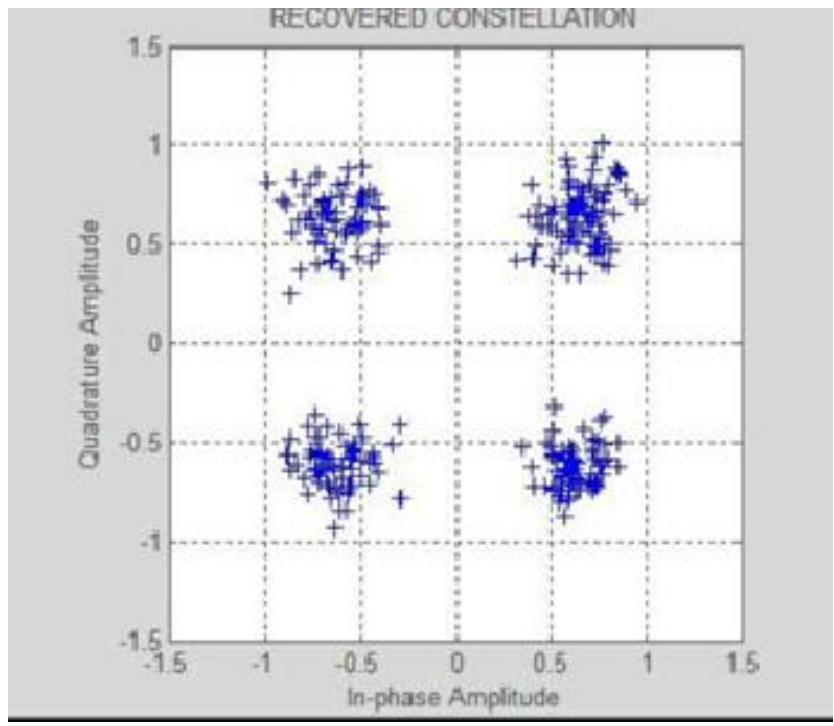

Fig. (8) shows the constellation diagram with use of the phase lock loop.

As shown in figure, the received constellation is getting in proper quadrant as per the transmitted quadrant.

\section{Eye diagram of the signal without phase lock loop}

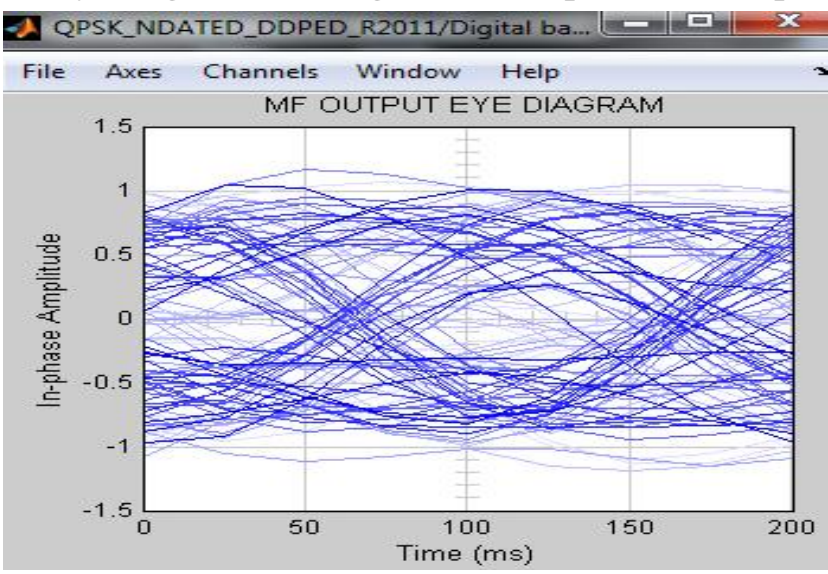

Fig. (9) a. Eye diagram of the signal without phase lock loop

Fig. (9) a. shows the eye diagram of the received signal without using the phase lock loop. As shown in diagram, the signal is scattered. This phase shift of the signal can't be distinguished and it shows the effect of Doppler shift that occurs in the communication.

\section{Eye diagram of the signal with phase lock loop}

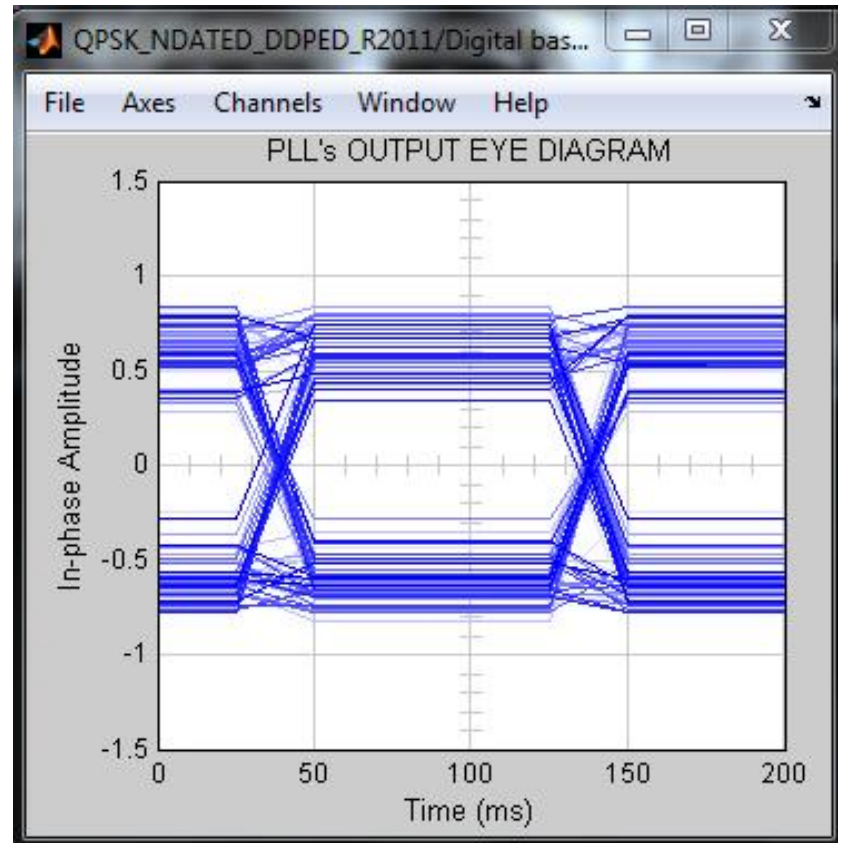

Fig. (9) b. Eye diagram of the signal with phase lock loop

Fig. (9) b. shows the eye diagram of the reviewed signal when phase lock loop is used. Phase lock loop provides a wideband acquisition to the signal because of which in receiver the signal in particular frequency band starting from $25 \mathrm{MHz}$ to $115 \mathrm{MHz}$ gets locked. And the result of this is shown in Fig. (9) b. Due to this phase lock loop the signal overcomes from the Doppler shift and in result to this; signal acquires the proper phase as shown in above Fig. (9) b

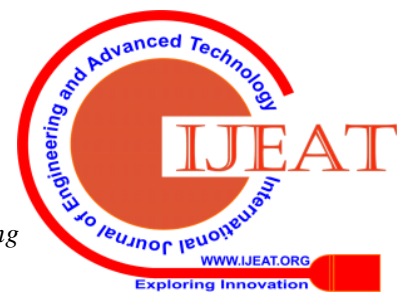




\section{QPSK Demodulator Based on Wideband Acquisition System}

\section{CONCLUSION}

The proposed wideband acquisition technique for QPSK demodulator is successfully designed and implemented with data rates up to $10 \mathrm{Mbps}$. The efficiency of the technique is validated with extensive simulations in MATLAB. The proposed technique can be implemented on field programmable gate arrays for the satellite testing on ground. The proposed technique achieves high resolution frequency estimation as compared to the existing PLL techniques.

\section{REFERENCES}

1. Akanksh Rai, Vaegae Naveen Kumar, "Wideband acquisition technique for QPSK demodulator", IEEE Transactions on Communications, May 20-21, 2016.

2. M R Raghavendra, S Sharada, K Chandrasekharam, Anshuman Sharma, Pooja Gupta, and Midhun M. "Design and Development of High Bit Rate QPSK Demodulator”, IEEE CONECCT2013.

3. Marvin K. Simon, "On the Bit-Error Probability of differentially encoded QPSK and offset QPSK in the presence of carrier synchronization”, IEEE Transactions on Communications, Vol. 54, No. 5, May 2006.

4. Robert. M. Gagliardi, "Satellite Communication", CBS, New Delhi, 1987. F. M. Gardner, Phase lock Techniques. John Wiley, 2005.

5. Marvin K. Simon, "On the Bit-Error Probability of differentially encoded QPSK and offset QPSK in the presence of carrier synchronization”, IEEE Transactions on Communications, Vol. 54, No. 5, May 2006

6. Spilkar JJ, "Digital Communication Satellite”, Prentice Hall, 1977.

7. Larry D. Paarmann, "Design and analysis of analog filters", Kluwer, Boston, 2001.

8. T. Nakagawa, K. Araki, "Effect of phase noise on RF communication Signals”, Proc. VTC 99-fall, 2000

9. M. Gasior, J. L. Gonzalez., "Improving FFT frequency measurement resolution by parabolic and Gaussian interpolation". European Organization for Nuclear Research, Switzerland, Feb 2004.

10. Application note, “Understanding FFT Windows". Lds-group.com, 2003.

11. Understanding FFTs and Windowing, NI tutorial. May 2015.

12. Satellite Terrestrial Cost Trade-off Study, Information Gatekeepers Inc.

\section{AUTHORS PROFILE}

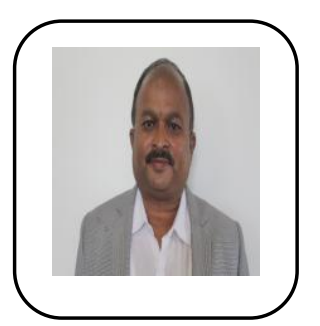

M. D. Kokate graduated in Electronics and Telecommunication Engineering from Govt Engineering Pune, MS, India and completed his post-graduation from Walchand College of Engineering Sangli,MS, India. Wireless Communication is research area andhis interest area is in signal processing, IoT and Data Sciences.

He has twenty nine years of Teaching and Industry experience. Currently he is working as Principal of SNJB's College of Engineering Chandwad,MS, India. He believes in developing effective teaching and learning processes in Engineering Education. He has written two Books and published many journal papers.

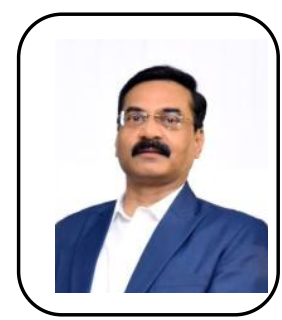

Dr. Abhay Wagh is The Director of Directorate of Technical Education of Maharashtra State. He received bachelor degree in Electronics Engineering, Gold Medalist Master of Engineering in Power Electronics and Ph.D. in Electronics and Instrumentation. He has 35 years working experience in the field of technical education as a teaching faculty, trainer, administrator and policy maker. He has worked as Director, MSBTE, Mumbai also worked as Deputy secretary to Government of Maharashtra in Higher and Technical Education Department, Mantralaya. He has worked a joint director, Dy. Director and Assistant Director in Directorate of Technical Education, Maharashtra.

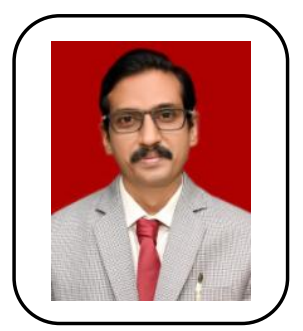

Dr. Wankhede Vishal Ashok is Principal of SNJBs S.H.H.J.B. Polytechnic Chandwad. He has received Bachelor degree in Electronics Engineering in 2000 from North Maharashtra University and Master of Engineering in 2007 from Babasaheb ambedkar Marathwada University. He has received Ph.D. in 2014 from Sardar Vallabhbhai National Institute of Technology. His major research interest is in the field of WiMAX, LTE, Cognitive Network, 5G technologies. He has published more than 30 international research papers in various International conferences and journals. 